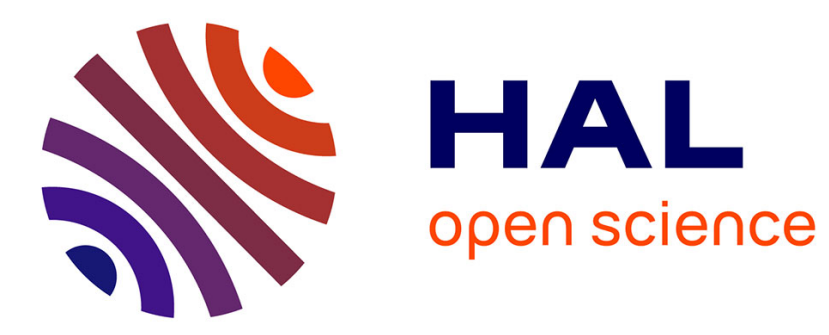

\title{
Lorsque l'industrie cinématographique sert de milieu au développement du jeu d'aventure: le cas de Maniac Mansion
}

Sébastien Genvo

\section{- To cite this version:}

Sébastien Genvo. Lorsque l'industrie cinématographique sert de milieu au développement du jeu d'aventure: le cas de Maniac Mansion. Décadrages, 2018, Jeu vidéo et cinéma, 39, pp.43-61. 10.4000/decadrages.1331 . hal-02900538

\section{HAL Id: hal-02900538 \\ https://hal.science/hal-02900538}

Submitted on 16 Jul 2020

HAL is a multi-disciplinary open access archive for the deposit and dissemination of scientific research documents, whether they are published or not. The documents may come from teaching and research institutions in France or abroad, or from public or private research centers.
L'archive ouverte pluridisciplinaire HAL, est destinée au dépôt et à la diffusion de documents scientifiques de niveau recherche, publiés ou non, émanant des établissements d'enseignement et de recherche français ou étrangers, des laboratoires publics ou privés. 


\title{
Lorsque l'industrie cinématographique sert de milieu au développement du jeu
} d'aventure : le cas de Maniac Mansion

\author{
Référence : Genvo, S., (2018). Lorsque l'industrie cinématographique sert de milieu au \\ développement du jeu d’aventure : le cas de Maniac Mansion, Revue Décadrage, 39 \\ Sébastien Genvo
}

Dans un article abordant les principales évolutions formelles des jeux d'aventure des années 1970 jusqu'à la fin des années 1990, Jonathan Lessard souligne que les transformations de ce genre, alors très populaire sur ordinateur personnel, se firent à partir de plusieurs référents culturels qui guidèrent les expérimentations et explorations des concepteurs de l'époque ${ }^{1}$. Si, à la naissance du genre, la métaphore majeure qui inspire ses premières mises en forme est celle du livre interactif (une grande partie de ces jeux étant alors uniquement en mode texte), un « tournant » narratif s'opère dans les années 1980 en suivant notamment une autre forme de métaphore, celle du film interactif. Pour étayer son analyse, Jonathan Lessard insiste notamment sur les changements de contextes technologiques qui ont guidé ces évolutions :

\begin{abstract}
La métaphore du livre interactif pour le jeu d'aventure apparaît dans le contexte des macroordinateurs industriels et de la première génération de microordinateurs. La majorité de ces machines sont conçues pour l'affichage de texte - exception faite de l'Apple II dont la diffusion est initialement limitée par son coût prohibitif -, ce qui facilite les parallèles avec l'imprimé. Ce contexte change au cours de la première moitié des années 1980. Désormais, les machines les plus répandues (Apple II, Commodore 64 et Atari 400/800) sont précisément conçues pour l'affichage graphique. $^{2}$
\end{abstract}

Cette analyse du rôle que joue le contexte technologique mais aussi culturel dans les évolutions des formes de jeu rejoint nos propres réflexions sur une approche deleuzienne des phénomènes ludiques $^{3}$. Selon nous, l'acte de jouer à un jeu procède d'un agencement entre un système de règles, des éléments fictionnels et un contexte pragmatique, où chaque agencement emprunte différents «fragments décodés » à son milieu (déterritorialisation) pour les « recoder» en territoire de jeu (reterritorialisation) :

\footnotetext{
1 Jonathan Lessard, «Livre, film ou monde interactif? Les métaphores de la construction formelle du jeu d'aventure », Mémoires $d u$ livre, vol. 5, $\mathrm{n}^{\circ}$ 2, printemps 2014. Disponible en ligne : https://www.erudit.org/fr/revues/memoires/2014-v5-n2-memoires01373/1024781 ar/.

${ }^{2}$ Idem, sans pagination.

${ }^{3}$ Sébastien Genvo, Penser la formation et les évolutions du jeu sur support numérique, Mémoire d'habilitation à diriger des recherches, Université de Lorraine, 2013. Disponible en ligne : http://www.ludologique.com.
} 
Le territoire est fait de fragments décodés de toutes sortes, empruntés aux milieux, mais qui acquièrent alors une valeur de propriétés ${ }^{4}$.

Pour Deleuze, les vecteurs de mutation, de variation, sont une des composantes de tout agencement, qui l'orientent vers un devenir.

Pas d'agencement sans territoire, territorialité, et re-territorialisation qui comprend toutes sortes d'artifices. Mais pas d'agencement non plus sans pointe de déterritorialisation, sans ligne de fuite, qui l'entraîne à de nouvelles créations, ou bien vers la mort ? ${ }^{5}$

Cette approche vise donc à souligner la nécessité de penser les jeux comme des phénomènes en perpétuelle transformation qui sont modelés par leur contexte environnant afin d'acquérir un sens ludique; en retour, chaque agencement de jeu a la capacité de générer de nouvelles pratiques et formes, dessinant de la sorte de nouvelles lignes de déterritorialisation. Dans notre perspective, un jeu vidéo ne peut s'instituer en un genre déterminé qu'à partir d'une homogénéisation de rapports de force : certains agencements vont, dans un contexte donné, paraitre plus typiques que d'autres de ce qu'on pense être un jeu de genre (cette perception pouvant évoluer). Il peut y avoir des périodes de stabilisation (où il y aura parfois simplement du neuf dans un genre donné) et des périodes de variations, voire de ruptures (des innovations donnant naissance à de nouveaux genres).

Maniac Mansion (Lucasfilm games, 1987) va servir de parangon dans l'histoire du jeu d'aventure pour stabiliser pendant un temps les contenus relevant de ce genre, cette homogénéisation s'effectuant à partir de « fragments » empruntés à un milieu spécifique, celui de l'industrie cinématographique. Cela participera notamment, comme l'a souligné Jonathan Lessard, à faire du film interactif l'une des principales métaphores guidant les évolutions formelles du genre au cours des années 1990. Pour présenter la façon dont cette œuvre vidéoludique a servi de socle à cette transformation, nous reviendrons tout d'abord sur la naissance du jeu d'aventure sur ordinateur individuel puis sur le «milieu » de développement du jeu offert par Lucasfilm Games. Nous analyserons ensuite ce qui, sur le plan du contenu, fait de Maniac Mansion une œuvre qui recode en territoire de jeu des fragments issus de son contexte cinématographique d'origine.

\section{Brève histoire de la naissance du jeu d'aventure}

À partir de l'étude d'un agencement de jeu initial, qui prend place dans un territoire donné, nous allons suivre certaines lignes de déterritorialisation et de re-territorialisation, ou vecteurs de

\footnotetext{
${ }^{4}$ Gilles Deleuze et Félix Guattari, Capitalisme et schizophrénie, Tome 2 : Mille plateaux, Paris, Éditions de Minuit, 1980 , p. 629.

${ }^{5}$ Gilles Deleuze, Claire Parnet, Dialogues, Paris, Flammarion, 1977, p. 84.
} 
mutations, qui l'ont traversé. Il ne s'agit donc pas de construire un lien artificiel de cause à effet entre une série d'événements, ces chaînes causales n'existant pas nécessairement au moment des faits. En effet, supposer la mise en œuvre d'une séquence orientée d'événements constitue une illusion rhétorique, cette orientation n'apparaissant pas nécessairement pour les acteurs de l'époque et ne tenant pas compte de la contingence, de l'aléa qui peut parfois participer à la formation des agencements. Plutôt que de voir des continuités, il faut donc être attentif aux ruptures qui s'effectuent entre plusieurs agencements, celles-ci participant à la création d'une identité propre. En somme, l'enjeu n'est pas de prêter au genre étudié certaines caractéristiques pour recenser rétrospectivement les jeux qui répondraient à ces critères, puisque ceux-ci ne sont jamais complètement fixés et stables, mais de comprendre comment à travers différents processus de déterritorialisation et de reterritorialisation des agencements de jeu ont été transformé par leurs milieux, entraînant des périodes de variation mais aussi de stabilisation. Ainsi, à propos d'Adventure (1976) de William Crowther, qui est souvent considéré comme le premier jeu d'aventure de l'histoire, Jonathan Lessard peut-il écrire :

Adventure est souvent appréhendé en termes d'événements subséquents, positionnés à l'origine d'une chaîne téléologique qui mène à des objets et sujets d'aujourd'hui. Bien sûr, ni William Crowther ni Don Woods n'ont jamais entendu parlé de jeux d'aventure, sans parler d'actionaventure ou de MMORPGs [Massively Multiplayer Online Role-Playing Games]. En d'autres termes, considérer Adventure comme un jeu d'aventure est quelque peu anachronique. Le simple concept de « jeux vidéo », tout comme la catégorie culturelle que nous connaissons aujourd'hui, étaient inconnus de ces programmeurs. ${ }^{6}$

Le contexte de conception d'Adventure joue un rôle essentiel dans la jouabilité et la thématique qui donnent forme à ce jeu. Il présente au moment de son développement un agencement original de mécanismes de jeux qui servit de socle commun à nombre d'autres jeux qui lui furent par la suite apparentés : l'exploration d'un monde fictionnel, la présence d'un récit d'événements dont l'actualisation dépend de la progression du joueur, la présence d'énigmes dont la résolution conditionne l'avancée. Comme on peut le constater, une logique d'exploration spatiale unit ces trois mécanismes. Dans Adventure, celle-ci se fait à travers des textes de description adressés aux joueurs (le jeu est uniquement en mode textuel), le joueur pouvant entrer des instructions simples de deux mots (verbe + complément) pour interagir avec l'environnement («go west», «take lamp»). Le contexte de développement permet de comprendre en grande partie ce qui guida l'adoption de ces choix de conception. Adventure a

\footnotetext{
${ }^{6}$ Jonathan Lessard, «Adventure Before Adventure Games. A New Look at Crowther and Woods's Seminal Program », Games and Culture, vol. 8, $\mathrm{n}^{\circ} 8$, février 2013, sans pagination. En ligne : http://journals.sagepub.com/doi/abs/10.1177/1555412012473364 [traduction de l'auteur].
} 
été initialement développé à partir de 1975 par William Crowther qui est membre d'une équipe de programmation dans une entreprise technologique de recherche et développement et participa entre autres au développement du réseau ARPANET (précurseur d'Internet). Mais Crowther avait aussi plusieurs hobbies qui expliquent les thématiques au centre du jeu. Il était en effet un spéléologue expérimenté et un amateur du jeu de rôles Donjons \& Dragons. Le logiciel que Crowther commence à développer en 1975 sur un macroordinateur de son entreprise propose d'explorer un système de grottes qui reproduit la topographie de cavernes dans le Kentucky que Crowther avait réellement explorées et cartographiées. Il y ajoute des trésors qu'il faut trouver et quelques éléments de fantasy. De même, l'adresse qui est faite au joueur par l'ordinateur en attente d'instructions fait penser au rôle du maître de jeu dans les jeux de rôles, qui lance fréquemment l'invective suivante aux participants : «que faitesvous? ». Les premiers utilisateurs de cette version d'Adventure furent les filles de Crowther et quelques collègues.

Comme l'indique l'anthropologue Thomas Malaby ${ }^{7}$, un jeu n'est pas à considérer comme une forme stable et immuable mais plutôt comme un processus toujours susceptible d'être modifié par ses pratiques. Ceci est particulièrement vrai d'Adventure. En 1976, Don Wood, un jeune hacker, étudiant à l'université de Stanford, met la main sur une copie du jeu et écrit à Crowther pour lui demander la permission d'étoffer son programme. Il renforça notamment les références faites à la fantasy, le genre littéraire de l'herö̈c fantasy étant particulièrement prisé par la communauté hacker. Cette version connaîtra une très grande fortune dans de nombreux campus américains en étant mis à disposition sur l'ARPANET. Cet agencement singulier de mécanismes ludiques fait particulièrement sens auprès de son public, essentiellement les chercheurs et étudiants appartenant à la communauté des hackers. Ces étudiants ingénieurs appréciaient particulièrement explorer les possibilités d'un système informatique, les détourner par la programmation dans un esprit ludique. Adventure était un jeu qui répondait tout à fait aux attentes des hackers concernant l'exploration d'un système et se prêtait très bien à leurs expérimentations. Selon Nick Montfort ${ }^{8}$, l'exploration du réseau de cavernes par l'utilisation d'un langage naturel générait un plaisir proche de la programmation : les programmeurs se battent avec un système qui ne fait ce que l'on veut que si l'on trouve la bonne façon de l'exprimer. La résolution des énigmes du jeu, qui permettaient de continuer l'exploration, se faisait en trouvant les bonnes instructions, en trouvant le mot juste et les combinaisons de mots

\footnotetext{
${ }^{7}$ Thomas Malaby, « Beyond Play. A New Approach to Games », Games and Culture, vol. 2, n 2, avril 2007, pp. 95-113.

${ }^{8}$ Nick Montfort, Twisty Little Passages : An Approach to Interactive Fiction, Cambridge, MIT Press, 2003.
} 
adéquates. On le voit, les fragments issus du milieu de conception du logiciel recodés en territoire de jeu sont notamment la programmation informatique, la spéléologie et les jeux de rôles.

Adventure eut un rôle déterminant dans la naissance d'une compagnie qui tiendra une place centrale dans la mise en forme du jeu d'aventure et dans sa diffusion à plus grande échelle : Sierra On-line, fondée par Roberta et Ken Williams. En 1979, Roberta découvre sur un ordinateur de son mari, Ken, une version d'Adventure. Le jeu la passionne et très vite elle veut en trouver d'autres semblables, mais à l'époque l'offre est plutôt mince. Elle décide donc de créer son propre jeu en persuadant son mari de l'aider à relever ce défi. C'est ainsi que Mystery House voit le jour en 1980 sur Apple II (fig. 1).

L'activité d'exploration spatiale et de résolution d'énigmes par commande textuelle de deux mots est toujours au cœur du jeu. Mystery House se détache cependant de son modèle par son cadre fictionnel. Le joueur entre dans une maison victorienne pour trouver un trésor caché (comme dans Adventure), mais il découvre très vite que les occupants de la maison sont tués au fur et à mesure de l'exploration. Le joueur doit affronter le meurtrier pour trouver le trésor et sortir de la demeure. En somme, la fiction s'écarte des romans de Tolkien, et se rapproche selon Ken Williams ${ }^{9}$ du film House on Haunted Hill (La Nuit de tous les mystères, William Castle, E.-U., 1959). L'espace domestique, qui est en train d'être investi comme lieu de jeu numérique à cette époque (par les consoles de salon et les premiers microordinateurs), est exploité à son tour comme sujet d'exploration ludique à travers le médium informatique. À ce titre, Roberta Williams transforme en jeu son espace de vie quotidien, qui devient dans Mystery House un lieu dangereux dont il faut s'échapper. Roberta Williams travaille sur le scénario de ses premiers jeux dans sa cuisine ${ }^{10}$, celle-ci constituant dans Mystery House une pièce essentielle où un mur friable peut être détruit pour mener au fameux trésor (des bijoux).

Mais l'originalité du logiciel tient surtout dans l'ajout, en plus des indications textuelles, d'une représentation graphique sommaire (quasi monochrome et sans animation) pour chaque lieu, présenté comme si la scène était perçue en ocularisation interne (à travers une vue « subjective »). Cette représentation n'est pas qu'une illustration mais comporte des éléments qui peuvent aider le joueur dans sa progression, tous les indices utiles ne figurant pas

\footnotetext{
9 J. Franck, «The Essential 100: No 78, Mystery House», 1UP.com, 2012, en ligne : http://www.1up.com/features/essential-78-mystery-house

${ }^{10}$ Un catalogue de Sierra On-Line de 1988 qui revient sur l'histoire de l'entreprise insiste à ce titre sur le lieu de travail initial de Roberta, en titrant Un miracle issu de la cuisine («A miracle from the kitchen »). En ligne : http://mocagh.org/sierra/sierra-88catalog2.pdf.
} 
uniquement dans la description textuelle. Le joueur doit trouver la dénomination correcte de l'objet représenté pour l'utiliser, bien que son identification soit parfois ardue au vu de la faible résolution (et qualité) des graphismes. Un carré dessiné sur un mur est par exemple un bouton sur lequel il faut appuyer pour ouvrir un passage secret.

Le succès fut immédiat et croissant, puisque le jeu se vendit à 10000 exemplaires, ce qui était très conséquent à une époque où le marché des jeux sur ordinateurs personnels était encore embryonnaire. Cela encouragea les Williams à donner davantage d'ampleur à leur entreprise en développant d'autres jeux du «genre ». Ils désignent ainsi leurs jeux sous le qualificatif d' « aventure haute résolution », faisant par là-même référence à leur modèle initial. À partir de ce moment, toute une série de jeux reprenant l'agencement de mécanismes au centre d'Adventure vont employer l'illustration graphique pour décrire l'environnement fictionnel. Le texte reste cependant central pour interagir mais aussi pour transmettre le récit.

Un tournant dans les productions de Sierra s'effectuera en 1984 avec King's Quest (fig. 2) qui est un jeu devant initialement démontrer les capacités techniques supérieures de la nouvelle machine d'IBM, le PC Jr.

Il est cette fois possible de diriger directement un personnage à l'écran, on peut agir dans l'image, introduisant par là même l'animation au sein des jeux d'aventure Sierra (les jeux précédents de l'éditeur reposant sur des images fixes). La partie graphique n'a plus pour rôle d'accompagner ou compléter le texte mais devient support d'exploration. Les modalités d'interaction sont néanmoins identiques aux productions précédentes de Sierra, puisqu'un analyseur syntaxique répond à des instructions en anglais faites de deux mots clés. Ce système d'interaction par commandes textuelles donne potentiellement une grande liberté d'interaction avec l'environnement fictionnel. L'une des difficultés posées par ce type de jouabilité est de trouver les mots reconnus par la machine, d'autant que dans King's Quest aucune description textuelle n'est donnée pour les éléments graphiques; le joueur doit donc deviner la dénomination exacte de certains éléments du décor : voit-on par exemple un « buisson » ou une «fougère »? Se tromper de mot empêchera toute action. Ce mode d'interaction, mêlant reconnaissance syntaxique et personnage animé dans un décor, sera employé dans l'ensemble des séries à succès de Sierra des années 1980. Des propositions et expérimentations différentes de jouabilité apparaissent néanmoins tout au long de cette décennie dans les jeux d'aventure. Un autre système d'interaction, fondé davantage sur l'action que sur l'image, va cependant peu à peu remplacer la commande textuelle à partir de la fin des années 1980, celui du pointer et cliquer (ou point \& click en anglais). Ce sera notamment Maniac Mansion qui va imposer ce 
nouveau standard dans le cadre du jeu d'aventure, l'orientant définitivement vers l'exploration d'une image animée, à l'inverse de son modèle initial qui était davantage comparé à la littérature interactive.

\section{L'entrée de Lucasfilm Games dans l'industrie vidéoludique}

Pour comprendre quelle sorte de milieu Lucasfilm Games offrait au développement de jeux vidéo, il faut revenir sur l'historique de sa maison mère, Lucasfilm, et sur les liens qu'entretenaient l'industrie du cinéma et celle des jeux vidéo au début des années 1980. Comme le rappelle Alexis Blanchet ${ }^{11}$, le secteur des jeux vidéo se constitue au début des années 1970 à une époque où le cinéma hollywoodien connaît la fin d'une longue période de déclin et une phase de mutation. Cette crise était notamment causée par la concurrence de la télévision depuis les années 1950 et par l'émergence de la contre-culture à la fin des années 1960, dont est notamment issu le phénomène hacker. C'est alors l'émergence du «New Hollywood» qui marque une réorganisation de la production cinématographique. Elle se traduit notamment par une prise de contrôle des firmes hollywoodiennes par de puissants groupes industriels qui vont former des conglomérats de médias, ce qui ouvre la voie à de nouveaux marchés et à d'autres formes d'expérimentation. Le nouvel Hollywood impose alors une nouvelle conception du film en salle, qui sert comme produit d'appel pour une valorisation marchande qui s'effectue sur d'autres supports. Il s'agit de considérer la sortie au cinéma comme événement qui crée le produit culturel, dont l'impact peut être réactivé à tout moment sur un nombre infini de médias.

Même si Lucasfilm ne faisait pas partie de ces conglomérats et pouvait être considéré comme une firme indépendante de ces «majors », il est indéniable que la stratégie commerciale adoptée par Georges Lucas épousait pleinement ces logiques de diversification. Elle allait même les développer et les intensifier :

C'est un peu en marge des conglomérats et à partir d'une production cinématographique, Star Wars (1977), que le réalisateur/producteur Georges Lucas engage une démarche industrielle, économique et esthétique, à proprement parler intermédiatique, en exploitant sous de nombreuses formes un même univers de fiction. [...] Star Wars pose à lui seul les jalons d'un rapport renouvelé de Hollywood au cinéma de divertissement et à ses possibilités multimédiatiques et financières ${ }^{12}$.

\footnotetext{
${ }^{11}$ Alexis Blanchet, Des pixels à Hollywood, Paris, Pix'n'love Editions, 2010.

${ }^{12}$ Idem, p. 115.
} 
En effet, comme le disent les fans, Star Wars c'est bien plus qu'un film ${ }^{13}$. Depuis sa naissance, c'est un univers fictionnel en expansion constante ${ }^{14}$ sur de nombreux supports et objets dérivés. Et pour mettre en œuvre la domination de cet empire, Lucas investit dans de nombreux secteurs, ce qui lui permet à la fois de maîtriser l'ensemble du processus de production et de diversifier ses activités. C'est ainsi qu'il fonde en 1975 Industrial Light \& Magic pour développer les effets visuels de Star Wars. Guidé par un désir d'innovation technique, Lucas décide d'étendre encore le champ de compétence de sa firme. Comme il le note lui-même, «mes rêves étaient de déplacer la réalisation de films au-delà de sa technologie inhérente au XIXe siècle pour la faire entrer dans l'ère de l'informatique ${ }^{15}$. Il met alors en place dès 1979 une division informatique qui va à la fois mener des activités de recherche et développement et rapporter de l'argent, lorsqu'il s'agit par exemple de développer l'«Effet Genesis» (produit à partir d'une technologie fractale) de Star Trek II : The Wrath of Khan (Nicholas Meyer, E.-U., 1982).

Un petit groupe consacré au développement de jeux fut rapidement recruté au sein de la division informatique, en étant libre d'émettre des idées de jeu. Lucas, toujours dans une optique d'innovation, souhaitait que ces projets présentent une certaine originalité et indépendance par rapport aux films produits par la compagnie, et il n'était donc pas question à ce moment de développer en interne une déclinaison de Star Wars (c'est Atari qui s'en chargera, en sortant en 1983 le premier jeu d'arcade dédié au film).

Néanmoins le contexte cinématographique offert par la maison mère allait indéniablement jouer sur la mise en forme des premières idées, comme le prouve une note rédigée par David Fox en 1982, qui est l'une des premières recrues de ce nouveau groupe. Il énumère plusieurs réflexions sur le développement de jeux et fait immédiatement le lien avec les films de Lucas :

\section{Comme notre programme de jeu sera un produit Lucasfilm, il semble approprié de porter un regard sur ce pour quoi Lucasfilm est célèbre : ses films. ${ }^{16}$}

Fox liste alors plusieurs éléments forts des épisodes de Star Wars (par exemple la présence d'une histoire qui est captivante « comme un livre que l'on ne peut poser »). Il s'interroge aussi sur la possibilité d'utiliser certains éléments qui rappellent ces productions cinématographiques. Dans sa note, il signale aussi plusieurs limitations des jeux informatiques qu'il faut dépasser. David Fox souligne notamment que davantage d'humour est nécessaire ou encore qu'un jeu

\footnotetext{
${ }^{13}$ Voir Laurent Jullier, Star Wars. Anatomie d'une saga, Paris, Armand Colin, 2005.

${ }^{14}$ Alain Boillat, Star wars : un monde en expansion, Chambéry, Editions ActuSF, 2014.

15 George Lucas, «Foreword», dans Rob Smith, Rogue Leaders: The Story of LucasArts, San Francisco, Chronicle Books, 2008, p. 7 [trad. de l'auteur].

${ }^{16}$ Idem, p. 14.
} 
comportant des animations graphiques exécutées de façon expertes peut le transformer en succès.

Le milieu de production offrait de la sorte un cadre favorable à l'émergence de jeux à dimension narrative (qui étaient alors essentiellement représentés par les jeux d'aventure) et une identité visuelle développée avec des références intermédiatiques marquées. En 1984, Peter Langston, qui fut le premier membre recruté par Lucas pour développer cette division, souligne d'ailleurs l'ambition de Lucasfilm Games :

Nous aimerions fournir des outils pour rendre la production de jeux plus facile. Ce serait comme faire des films, où quelqu'un qui est un bon narrateur pourrait trouver quelqu'un qui connaît vraiment le code 6502 [langage de programmation de l'époque]. Georges Lucas est un grand narrateur - nous aimerions être capables de le mettre dans un environnement où il pourrait transformer ses visions en jeux vidéo. ${ }^{17}$

Le premier jeu d'aventure de Lucasfilm Games se fera en 1986 avec l'adaptation d'un film dont Lucas est producteur, Labyrinth, réalisé par Jim Henson (le film repose en grande partie sur l'utilisation de marionnettes, avec la présence de David Bowie en « méchant » de l'histoire). L'introduction du jeu reprend l'interface usuelle d'un jeu d'aventure uniquement en mode texte, sans interface graphique. Une série de descriptions textuelles indique au joueur qu'il se trouve devant un cinéma pour aller voir le film Labyrinth. L'interface de commande est cependant différente et ne repose plus sur le fait de trouver le mot juste pour interagir. Les actions disponibles sont en effet listées dans un menu déroulant en bas de l'écran et une fois choisies elles peuvent être employées sur une liste de mots qui dépend de l'inventaire du personnage et de sa position dans l'espace diégétique (par exemple, s'il est proche du stand de popcorn, il peut donner un dollar) (fig. 3).

Une fois le personnage installé dans la salle, l'interface textuelle laisse place à un carton dessiné sur le modèle de ceux du cinéma muet (fig. 4), indiquant que le film commence, puis à une image animée de David Bowie dans un écran de cinéma (fig. 5), qui s'adresse directement au personnage incarné par le joueur (tout comme dans La Rose pourpre du Caire de Woody Allen, 1985). Il lui indique qu'il va être emprisonné dans le « labyrinthe » et ne pourra en sortir que lorsqu'il trouvera et vaincra son propriétaire.

Le joueur dirige ensuite un personnage dans un décor graphique, tout comme dans King's Quest, si ce n'est que l'écran défile lorsqu'il se déplace à droite ou à gauche (pour reprendre le terme technique consacré, un scrolling suit les déplacements du héros, voir figure 6). Des portes

17 Peter Langston cité dans NewsWeek, «Lucas looks beyond film», 1984, p. 12, en ligne : http://www.langston.com/LFGames/NewsweekAccess1984article.html [trad. de l'auteur]. 
lui permettent de changer d'écran. L'interface de commande reste toutefois la même que celle mise en place durant la séquence en mode texte (sur le mode du menu de mots défilant).

En somme, à travers la mise en scène de l'introduction du jeu en mode texte, Labyrinth affiche l'ambition de Lucasfilm Games : faire évoluer la jouabilité du jeu d'aventure en la détachant de son modèle initial (le jeu d'aventure textuel) pour l'ancrer dans celui de l'image animée, avec un cadre de référence fictionnel issu du cinéma. En somme Lucasfilm Games permet une reterritorialisation du jeu d'aventure dans un milieu qui offrira de nouveaux fragments aux agencements ludiques qui vont en émerger (sans qu'il n'y ait cependant tout à fait rupture, ce qui permet de garder une continuité générique). Ce n'est cependant pas Labyrinth qui servira de modèle pour l'uniformisation et la stabilisation du genre pendant plusieurs années, mais son successeur, Maniac Mansion.

\section{Le jeu d'aventure comme image actée}

Maniac Mansion, paru initialement sur Commodore 64 en 1987, naît de la rencontre de deux employés de Lucasfilm Games : Ron Gilbert et Gary Winnick. Ron Gilbert a été recruté en 1985 à 21 ans dans la firme de Georges Lucas, en tant que programmeur pour Commodore 64, où il effectue au départ l'adaptation des premiers jeux de la firme pour différents ordinateurs. À son adolescence, Ron Gilbert a d'ailleurs été fortement marqué par Star Wars, au point de réaliser en Super- 8 avec des amis quelques films inspirés de cet univers. Étudiant, il découvre les jeux d'aventure textuels, dont Adventure. Lorsque Gilbert est recruté en 1985, Gary Winnick travaille sur Labyrinth. Il est le premier graphiste à avoir été recruté par Lucasfilm Games. Auparavant, il a entre autres travaillé chez Continuity Associates, un studio d'art et d'illustrations formé par les cartoonists Neal Adams et Dick Giordano (il s'agit d'un studio de sous-traitance qui a notamment travaillé pour Marvel Comics). C'est à la fois la culture des comics américains mais aussi des films pour adolescents du début des années 1980 qui vont servir de sources d'inspiration, à l'instar d'un film d'horreur comme Creepshow (George A. Romero, E.-U., 1982), qui est lui-même un hommage aux comics d'horreur des années 1950 pour adolescents, comme Tales from the Crypt (série de comics créée par William Gaines et Al Feldstein et publiée entre 1950 et 1955). L'ambition des deux collègues est alors de créer des situations loufoques ou incongrues se déroulant dans des situations inspirées de ces références fictionnelles.

Maniac Mansion commence par la chute d'une météorite près de la maison d'un scientifique, le Docteur Ed, qui va alors devenir fou et capturer des adolescents pour les enfermer dans une 
machine destinée à aspirer leur cerveau, ceci sous le contrôle du météore, qui est une entité vivante. La petite amie du héros (Dave) est kidnappée et celui-ci décide de partir avec deux autres amis à l'exploration de la demeure du savant fou pour la libérer. Tout comme dans Mystery House, le jeu commence à l'extérieur d'une demeure dans laquelle le joueur doit pénétrer pour en révéler les secrets (et tout comme dans le premier jeu de Sierra, la clé de l'aventure se trouvera dans le sous-sol). Au début du jeu, le joueur peut choisir deux personnages supplémentaires au sein de six adolescents qui sont tous des stéréotypes d'adolescents des années 1980 (fig. 7). Il y a par exemple Bernard, qui est le type même du «geek» à lunettes féru d'informatique, Jeff le surfeur blond, Razor la punkette, ou encore Wendy qui veut devenir une romancière reconnue. Durant son aventure, le joueur pourra diriger les trois personnages. Les énigmes, de même que la façon de finir le jeu et les multiples fins possibles de l'histoire, seront différentes selon la combinaison choisie. Une veille radio peut par exemple être réparée, mais cela n'est possible que si le joueur a choisi Bernard dans son équipe. Chaque personnage représente ainsi une compétence singulière.

Tout comme dans King's Quest, le joueur déplace ses personnages au sein d'un environnement graphique (fig. 8). Le principe de combinaisons d'objets trouvés avec des éléments du décor reste toujours valable mais l'interface emploie à présent le principe du « pointer / cliquer » : une interface en bas de l'écran liste un ensemble de verbes utiles (ouvrir / prendre / donner /...) ; le joueur clique alors sur le verbe et un élément du décor pour interagir ; il n'y a plus dès lors de commande textuelle à entrer ou de termes justes à trouver pour interagir.

Pour Ron Gilbert, il s'agissait de s'écarter de l'interface textuelle présente dans King's Quest, qui impliquait de deviner les mots justes pour interagir avec les éléments du décor. Il n'est donc plus nécessaire de trouver la terminologie exacte pour pouvoir agir. Cela permet au joueur de se dispenser de clavier (il est possible de pointer et cliquer à l'aide d'un joystick ou d'une souris), ce qui permettra par ailleurs une adaptation du jeu dans de nombreux autres contextes, à travers notamment une version sur la console de Nintendo, la NES. Le contenu textuel étant également moins conséquent et moins central dans les modalités d'interaction par rapport aux autres jeux d'aventure, la traduction en d'autres langues est cette fois facilement envisageable.

En se détachant de la reconnaissance textuelle au profit de la logique du «point \& click», Maniac Mansion minimise le mécanisme initial des jeux textuels qui rapprochait l'activité du joueur de celui de la programmation. Bien sûr, il faut toujours trouver une combinaison de mots et un ordre d'instruction correct pour progresser (la phrase constituée par l'ordre verbe + objet est toujours écrite à l'écran), mais le nombre d'instructions possibles est plus limité, du fait que 
l'ensemble des verbes envisageables est listé (pour rappel, on active les verbes en cliquant dessus puis en pointant l'élément graphique du décor sur lequel on souhaite agir). En revanche, l'activité de découverte spatiale se trouve renforcée, en se doublant d'une activité d'exploration visuelle. Si à la naissance du jeu d'aventure graphique, avec Mystery House, l'image était un vecteur de description et le moteur principal de l'action restait le texte, avec Maniac Mansion, l'image devient pleinement le support de l'action, cette transition ayant été initiée par King's Quest. Dans Maniac Mansion, pour pouvoir agir, le joueur doit explorer l'image avec son curseur et repérer dans la représentation graphique les éléments qui peuvent servir à l'action. De même, à la différence des jeux d'aventure graphique développés jusqu'alors où chaque changement de lieu impliquait un changement d'écran (à l'exception de Labyrinth qui servait en quelque sorte de prototype sur ce point), Maniac Mansion met également en place un système de défilement, ou « scrolling » qui suit le déplacement du personnage, ce qui incite au déplacement dans l'image : on n'est jamais sûr que toutes les données utiles sont visuellement présentes. Explorer une image pour découvrir une histoire rapproche sur le plan formel le jeu d'aventure des comics, ce qui était une autre source d'inspiration pour Ron Gilbert et Gary Winnick.

Il n'y a quasiment plus de textes de description, seuls les personnages parlent entre eux ou s'adressent au joueur lorsqu'une action n'est pas possible ou lorsqu'un commentaire sur la situation est requis. À ce titre, Maniac Mansion introduit dans le jeu vidéo une convention narrative qui sera amenée à se répandre par la suite pour présenter des éléments de récit non jouables, celle de la «cut-scene » (ou scène de coupe), terminologie empruntée au cinéma ${ }^{18}$. Ce terme apparaît tel quel en tant que fonction dans le script de programmation de Ron Gilbert ${ }^{19}$ et désigne un moment du jeu où le joueur ne peut plus agir et passe à l'état de spectateur face à une série d'événements préétablis (la liste des verbes cliquables disparaît d'ailleurs à ce moment pour signifier au joueur qu'il ne peut plus agir, voir fig. 9). Dans le cas de Maniac Mansion, les cut-scenes ont essentiellement pour rôle de délivrer au joueur certaines informations permettant de faire avancer la narration en parallèle de ses actions et/ou de lui donner des indices sur les énigmes à résoudre. Plusieurs cut-scenes reviennent notamment sur les agissements des résidents de la demeure et leurs occupations quotidiennes.

\footnotetext{
${ }^{18}$ Ron Gilbert ne savait pas comment appeler ce genre de séquence qui lui rappelait les écrans entre les niveaux dans Ms. Pacman (Midway, 1981). Il demanda alors à Steve Arnold, président de Lucasfilm Games, quel était le nom pour ce genre de scènes qui coupaient de l'histoire principale dans les films, ce à quoi celui-ci lui répondit « cut-scenes ».

${ }^{19}$ Voir sa conférence à la Game Developer Conference 2011, où Ron Gilbert dévoile certains passages du script d'origine du jeu : http://www.gdcvault.com/play/1014732/Classic-Game-Postmortem-MANIAC.
} 
Comme on l'a vu, le principe de présenter une narration par l'intermédiaire d'écrans sur lequel le joueur ne pouvait agir n'était pas nouveau (il y avait par exemple des « cartons » textuels dans Labyrinth), mais le plus souvent l'apparition de ces écrans dépendait de la fin d'une séquence de jeu (fin de niveau, début et fin de jeu, changement de lieu, etc.). Dans Maniac Mansion, le déclenchement des cut-scenes est tout à fait indépendant de la fin ou du début d'une séquence et des agissements du joueur. Il peut ainsi arriver à tout moment, peu importe l'avancée du joueur dans sa découverte des lieux et dans sa résolution de l'énigme principale. Cela génère en quelque sorte une histoire parallèle à celle déclenchée par les actions du joueur (« l'histoire principale »), ce qui donne le sentiment que la maison vit à son propre rythme alors que le joueur s'introduit dans la propriété du Docteur Ed.

Les références cinématographiques qui nourrissent l'univers diégétique de Maniac Mansion ont à ce titre fortement nourri la réception du jeu. Le magazine ZZAP !64 (spécialisé dans les tests de jeux sur ordinateurs Commodore) de décembre 1987 insiste sur l'« atmosphère filmique » du jeu en le comparant à The Rocky Horror Picture Show (Jim Sharman, Angleterre/Etats-Unis, 1975) et à Scooby-Doo (Hanna-Barbera Productions, 1969-1994). Pour Commodore User ${ }^{20}$, le jeu rassemble des éléments de Psychose de Hitchcock (États-Unis, 1960), de Vendredi 13 (Sean S. Cunningham, Etats-Unis, 1980), de la Famille Addams et du Rocky Horror Picture Show. La référence récurrente à Rocky Horror Picture Show traduit aussi la perception des sous-entendus obscènes présents dans le jeu, qui mêle, tout comme dans le film de Jim Sharman (mais de façon moins prononcée), le sexe (à travers le personnage de la femme du docteur Ed qui est une infirmière lubrique), l'humour et l'horreur. Il est intéressant de constater que la référence aux films d'horreur et de série $\mathrm{B}$ se justifie à la fois à travers l'histoire et la diégèse véhiculées par le jeu, mais aussi à travers la jouabilité, notamment par la possibilité de diriger séparément trois protagonistes : «Cette méthode de contrôle individuelle permet au groupe de se séparer dans la tradition des films de série B, pour explorer la demeure ${ }^{21}$. Lucasfilm Games met donc bien en forme à travers ses jeux d'aventure des éléments différents que ceux qui ont permis l'avènement des premières aventures informatiques.

Si les références faites aux films (notamment d'horreur) et l'impression de jouer une histoire sont mises en avant par les critiques, les choix de game design et le positionnement adopté par l'équipe de Ron Gilbert par rapport aux jeux d'aventure textuels font aussi l'objet de louanges. Ainsi peut-on lire dans le numéro de février 1990 d'Amiga Computing, « bien que ce soit

\footnotetext{
${ }^{20}$ Bill Scolding, « Maniac Mansion », Commodore User, ${ }^{\circ}$ 51, 1987, p. 41.

21 « Maniac Mansion », Zzap64 !, 1987, p. 32.
} 
essentiellement une aventure, Lucasfilm a réussi à nous dispenser de toute cette écriture textuelle fastidieuse, et a réussi à créer un jeu animé qui est fun à jouer et plaisant à regarder $»^{22}$. En ce sens, Commodore Magazine ne manque pas de voir dans Maniac Mansion une évolution par rapport à la première incursion de Lucasfilm dans le jeu d'aventure, tout en faisant un parallèle avec la franchise phare de Sierra On-line :

L'année dernière Labyrinth a introduit des graphismes animés, en 3D, dans les aventures pour le [Commodore] 64, ce qui est habituellement réservé aux détenteurs d'Amiga qui peuvent jouer à des jeux Sierra comme la série des King's Quest. Dans l'ensemble de ses aspects, à l'exception de l'interface textuelle qui ressemblait à une roue, le jeu était de grande qualité. La seconde aventure de Lucasfilm Games, Maniac Mansion est encore meilleure, offrant une interface très pratique et quelques innovations inattendues ${ }^{23}$.

Outre l'interface, c'est aussi la gestion des animations et des personnages qui retient l'attention du testeur, qui voit dans le jeu des améliorations par rapport à la franchise phare des époux Williams (ce qui marque le début d'une longue concurrence entre les deux entreprises sur le terrain du jeu d'aventure). L'ensemble des jeux d'aventure de Lucasfilm Games dans les années 1980 et au début des années 1990 allait alors reprendre la jouabilité mise en œuvre par Maniac Mansion. Face à cette concurrence, Sierra On-Line abandonnera l'interface de commande textuelle de ses jeux, l'épisode 5 de King's Quest paru en 1990 étant le premier de cette série à adopter une interface semblable à celle des jeux Lucasfilm Games.

\section{Vers la stabilisation d'un modèle}

Que ce soit par la forme ou le fond, les choix de conception effectués dans Maniac Mansion rapprochent le jeu d'aventure du cinéma et l'éloignent de la littérature. C'est d'ailleurs la raison pour laquelle de nombreux magazines de l'époque insistent sur la dimension visuelle, animée et agie du jeu. Dans les années 1990, avec l'avènement des capacités de stockage plus importante du cédérom, de nombreux jeux d'aventure auront d'ailleurs recours à l'incrustation d'acteurs digitalisés et de « séquences cinématiques » (dont la notion de cut-scene de Ron Gilbert était annonciatrice) tout en conservant le principe du point \& click imposé par Maniac Mansion. Sierra multipliera notamment ces jeux mettant en scène des acteurs réels avec la série Phantasmagoria (1995, 1996) ou le second opus de sa série Gabriel Knight (1995).

\footnotetext{
${ }^{22}$ Mat Broomfield, « Maniac Mansion », Amiga Computing, n 21, 1990, p. 34 [trad. de l'auteur].

${ }^{23}$ Shay Addams, « Animated adventuring in Maniac Mansion », Commodore Magazine, $\mathrm{n}^{\circ}$ 12, 1987, p. 48 [trad. de l'auteur].
} 
En somme, ce que nous montre Maniac Mansion quant à son impact culturel dans le paysage vidéoludique, c'est qu'il a participé à changer le rapport au jeu d'aventure informatique en consacrant l'avènement d'une image actée ${ }^{24}$ au détriment d'une fiction interagie par l'écrit : l'image devient une entité visuelle malléable ouverte à une potentialité évolutive, où la narration repose sur «l'action faite par soi-même et pour soi-même sur la base de configurations narratives préréglées $»^{25}$. Dans Maniac Mansion, la narration se fait interactive par l'exploration d'un espace visuel, cette sensation étant d'ailleurs renforcée par l'introduction du scrolling. Comme le dit un fan, « vous ne savez jamais ce qui vous attend au tournant, et n'importe quoi peut, et va arriver ${ }^{26}$. Le terme «aventure » renvoyant également à ce qui doit advenir (adventura), ce joueur nous montre que Maniac Mansion nous fait bien vivre ici une aventure au sens quasi étymologique du terme.

\footnotetext{
${ }^{24}$ Pierre Barboza et Jean-Louis Weissberg (éd.), L’image actée. Scénarisations numériques, parcours $d u$ séminaire, Paris, L'Harmattan, 2006.

${ }^{25}$ Voir Etienne Armand Amato, « Interactivité d'accomplissement et de réception dans un jeu tridimensionnel : de l'image actée à l'image interagie», dans Pierre Barboza et Jean-Louis Weissberg (éd.), L'image actée. Scénarisations numériques, parcours du séminaire, op. cit., p. 159.

${ }^{26}$ www.maniacmansionfan.50webs.com/waystolose.html
} 